\title{
Safety of subcutaneous adrenaline as prophylaxis against acute adverse reactions to anti-venom serum in snakebite
}

\author{
A S Dassanayake ${ }^{1}$, P Karunanayake ${ }^{2}$, K T A A Kasturiratne ${ }^{1}$, M M D Fonseka ${ }^{1}$, B Wijesiriwardena ${ }^{2}$, S B Gunatilake ${ }^{1}$ \\ and $\mathbf{H} \mathbf{J}$ de Silva ${ }^{1}$
}

(Index words: Pulse rate, blood pressure, subcutaneous haematoma, cerebral haemorrhage)

\begin{abstract}
Objectives To study the safety of low dose subcutaneous adrenaline given as prophylaxis against acute adverse reactions to anti-venom serum (AVS) in patients bitten by snakes.

Methods Patients admitted with snakebite envenoming who satisfied inclusion criteria were given $0.25 \mathrm{ml}$ of 1:1000 adrenaline subcutaneously immediately before administration of AVS. They were observed for adverse effects, and pulse and blood pressure (BP) were monitored.

Results 51 patients [35 males, mean age 34.8 years (SD 14)] were included in the study. Adverse reactions to AVS occurred in $15(29.4 \%)$ patients. There was one death from suspected cerebral haemorrhage, and $3(5.9 \%)$ patients developed small haematomas at the subcutaneous injection site. There were no significant changes in mean pulse or BP following administration of subcutaneous adrenaline.

Conclusions Low dose subcutaneous adrenaline did not cause significant changes in pulse rate or BP. Although the death was unlikely to be directly related to subcutaneous adrenaline, we suggest further studies on the safety of this prophylactic treatment before its routine use.
\end{abstract}

\section{Introduction}

Antivenom serum (AVS) is the only effective treatnent for snakebite envenoming. Adverse effects of AVS are common, and include anaphylaxis. On average, about half the patients who receive AVS available in Sri Lanka develop adverse reactions to it $(1,2,3)$ and reaction rates as high as $68 \%$ have been reported (4). We recently showed that low dose adrenaline given subcutaneously immediately before administration of AVS to snakebite victims significantly reduces incidence of such acute adverse reactions (1). Its use in this manner has now been recommended in the Cochrane database (5). A major concern regarding use of adrenaline is the risk of intracerebral haemorrhage (6), a result of coagulopathy induced by some snake venoms and hypertension produced by adrenaline. In the study mentioned above (1), no significant adverse effects attributable to adrenaline were reported, but patients who were at high risk for adverse effects from adrenaline were excluded. Adrenaline given intravenously causes hypertension. Although this does not seem to occur with subcutaneous administration (7), fatal intracranial bleeding has been reported (8). The objective of this study was to further assess the safety of low dose subcutaneous adrenaline.

\section{Methods}

We prospectively studied consecutive patients with snakebite envenoming at the Colombo North Teaching Hospital, from April 2000 to October 2001. After informed consent, patients who satisfied inclusion criteria were given $0.25 \mathrm{ml}$ of 1:1000 adrenaline subcutaneously into the forearm immediately before administration of polyspecific AVS (Haffkine Laboratories, Mumbai, India) by intravenous infusion. Patients were excluded if they were pregnant, aged over 70 years, had hypertension, ischaemic heart disease, arrhythmias or cerebrovascular disease. They were observed for adverse effects, and pulse and blood pressure (BP) were recorded before, and every $5 \mathrm{~min}$ for $20 \mathrm{~min}$, and every $10 \mathrm{~min}$ for up to $60 \mathrm{~min}$ after adrenaline was given. In patients who developed adverse reactions to AVS, subsequent pulse and BP measurements were not included for analysis as these values were likely to be influenced by the reaction. Ethical approval was obtained for our study from the Ethics Committee, University of Kelaniya.

\section{Results}

Fifty one patients $[\mathrm{M}: \mathrm{F}=35: 16$; mean age 34.8 years (SD14)] with systemic envenoming following snakebite were included in the study. The offending snake was identified in $21(41.2 \%)$. Compared to pretreatment values, no significant changes in mean pulse rate [pretreatment $87.7 / \mathrm{min}$ (SD13.6)], systolic [pretreatment $120.1 \mathrm{mmHg}$ (SD13.2)] or diastolic [pretreatment $78 \mathrm{mmHg}$ (SD8.5)] BP were observed following administration of subcutaneous adrenaline (Student-t test) (Figure). Adverse reaction to AVS occurred in 15 (29.4\%) patients; they were severe in 3. There were $4(7.8 \%)$ other complications, one death and 3 $(5.9 \%)$ small haematomas at the subcutaneous injection site in the patients with coagulopathy.

The patient who died was a 65 -year old man, bitten by unidentified snake. On admission there was swelling at the site of bite (right foot) and coagulopathy (whole blood

${ }^{1}$ Faculty of Medicine, University of Kelaniya and ${ }^{2}$ Colombo North Teaching Hospital, Ragama.(Correspondence: H J de S, Faculty of Medicine, University of Kelaniya, P O Box 6, Ragama. Fax: 01 958337; e-mail: hjdes@ sltnet.lk. Submitted 26 March 2002, accepted 1 April 2002). 
clotting time $>20 \mathrm{~min}$ ). He was given subcutaneous adenaline followed by 10 vials of AVS. He developed a reaction to AVS (severe rigors, and tightening sensation in his throat and chest) $55 \mathrm{~min}$ after the infusion was started.

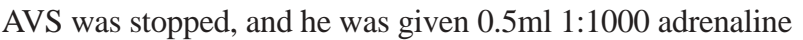
intramuscularly, $200 \mathrm{mg}$ hydrocortisone intravenously and $25 \mathrm{mg}$ promethazine intramuscularly. He recovered, and as the clotting time was less than 10 minutes by that time, no further AVS was given. About 10 hours later he became drowsy and unresponsive. His limbs were flaccid, tendon reflexes were present and plantar responses were extensor bilaterally. There was no neck stiffness. Optic fundi could not be visualised because of cataracts. Intracerebral haemorrhage was suspected, and as facilities for CT scan were not available in our hopsital, he was transferred to the National Hospital, Colombo. He died before CT could be performed. His relatives did not consent to post-mortem examination.

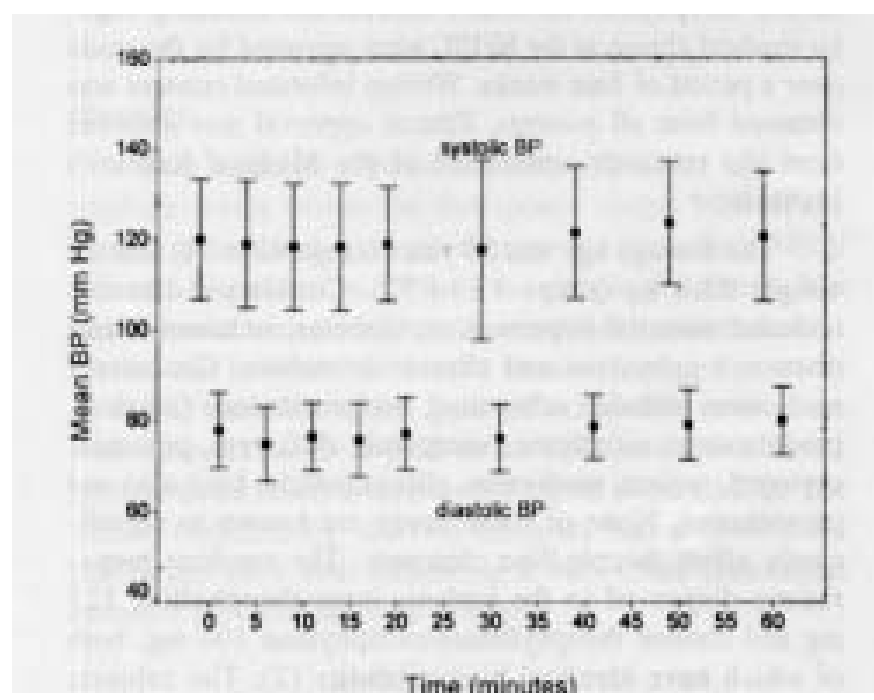

Figure. Mean systolic and diastolic blood pressures of patients who received low dose subcutaneous adrenaline. Vertical bars denote one standard deviation.

\section{Comment}

Low dose subcutaneous adrenaline did not cause significant changes in mean pulse rate or BP in our patients. This is in agreement with previous findings (7). The subcutaneous haematomas can be attributed to the injection. The patient who died, in addition to receiving subcutaneous adenaline about 11 hours before death, had snake venom induced coagulopathy, and developed a reaction to AVS which was treated with intramuscular adrenaline about one hour after the subutaneous dose. A recent study suggests that the half-life of adrenaline after subcutane- ous administration is $43 \mathrm{~min}$ (9). Based on this it is likely that almost all of the drug would be eliminated from the body 4 hours after administration, and that effects would have worn off by then, perhaps sooner. In another study (10), plasma adrenaline concentrations fell by 3 hours after subcutaneous administration, and effects lasted $90 \mathrm{~min}$. These findings make the death of our patient unlikely to be directly related to subcutaneous adrenaline. Nevertheless, further studies on the safety of prophylactic adrenaline shoud be performed before its routine use can be recommended in snakebite.

\section{References}

1. Premawardhena AP, de Sliva CE, Fonseka MMD, Gunatilake, SB, de Silva HJ. Low dose subcutaneous adrenaline to prevent acute adverse reactions to antivenom serum in people bitten by snakes: randomised placebo controlled trial. British Medical Journal 1999;318: 730-3.

2. Seneviratne SL, Opanayake CJ, Ratnayake NSL, Sarath Kumara $\mathrm{KE}$, Sugathadasa AM, et al. Use of antivenom serum in snake bite: a prospective study of hospital practice in the Gampaha district. Ceylon Medical Journal 2000; 45: 65-8.

3. Theakston RDG, Phillips RE, Warrell DA, Galagedara Y, Abeysekea DTDJ, et al. Envenoming by the common Krait and Sri Lankan cobra; efficacy and complications of therapy with Haffkine antivenom. Transactions of the Royal Society of Tropical Medicine and Hygiene 1990; 84: 301-8.

4. Kularatne SAM. Reactions to snake venom antisera: study of pattern, severity and management at General Hospital Anuradhapura. Sri Lanka Journal of Medicine 2000; 9: 8-13.

5. Nuchprayoon I, Garner P. Interventions for preventing reactions to snake antivenom. Cochrane Database Systems Review 2000: (2): CD002153

6. Khanna R, Hawkins WJ. A plea for caution in the use of adrenaline. British Medical Journal (electronic version); bmj.com, 19 May, 1999.

7. Heliborn H, Hjemdhal P, Daleskog M, Adamsson U. Comparison of subcutaneous injection and high dose inhalation of epinephrine - implications for self treatment to prevent anaphylaxis. Journal of Allergy and Clinical Immunology 1986; 78: 1174-9.

8. Horowitz BZ, Jadallah S, Derlet RW. Fatal intracranical bleeding associated with prehospital use of epinephrine. Annals of Emergency Medicine 1996; 28: 725-7.

9. Simons FE, Roberts JR. Gu X, Simons KJ. Epinephrine absorption in children with a history of anaphylaxis. Journal of Allergy and Clinical Immunology 1998; 101: 33-7.

10. Dahlof C, Mellstrand T, Svedmyr N. Systemic absorption of adrenaline after aerosol, eye-drop and subcutaneous administration to healthy volunteers. Allergy 1987; 42: 215-21. 